Review Article

\title{
Emergence of Convolutional Neural Network in Future Medicine: Why and How. A Review on Brain Tumor Segmentation
}

\author{
Behrouz AlIZADEH SAVAREH ${ }^{1}$, Hassan EMAMI ${ }^{2, a}$, Mohamadreza HAJIABADI ${ }^{3,4}$, Mahyar GHAFOORI ${ }^{5}$, Seyed MAJID \\ $\mathrm{AZIMI}^{6}$ \\ ${ }^{I}$ School of Allied Medical Sciences, Shahid Beheshti University of Medical Sciences, Tehran, Iran \\ ${ }^{2}$ Health Information Technology and Management department, Faculty of Paramedical Sciences, Shahid Beheshti University \\ of Medical Sciences, Tehran, Iran \\ ${ }^{3}$ Brain and Spinal Cord Injury Research Center, Neuroscience Institute, Tehran University of Medical Sciences, Tehran, Iran \\ ${ }^{4}$ Department of Neurosurgery, Shariati Hospital, Tehran University of Medical Sciences, Tehran, Iran \\ ${ }^{5}$ Department of Radiology, Hazrat Rasoul Akram Hospital, School of Medicine, Iran University of Medical Sciences, Tehran, \\ Iran \\ ${ }^{6}$ Chair of Remote Sensing Technology, Technical university of Munich, Germany \\ ${ }^{a}$ E-mail address: haemami1337@yahoo.com
}

(received 31 October 2017; revised 26 December 2017; accepted 1 February 2018)

\begin{abstract}
Manual analysis of brain tumors magnetic resonance images is usually accompanied by some problem. Several techniques have been proposed for the brain tumor segmentation. This study will be focused on searching popular databases for related studies, theoretical and practical aspects of Convolutional Neural Network surveyed in brain tumor segmentation. Based on our findings, details about related studies including the datasets used, evaluation parameters, preferred architectures and complementary steps analyzed. Deep learning as a revolutionary idea in image processing, achieved brilliant results in brain tumor segmentation too. This can be continuing until the next revolutionary idea emerging.
\end{abstract}

Key words: convolution neural network; brain tumor; segmentation; deep learning.

\section{Introduction}

The brain tumor is generated by abnormal growth of pathologic cells in the brain [1-3]. Due to the brain's role in the body, the people who are affected by tumors may have a serious dysfunction in sensation, movement, equilibrium, cognition [2, 4-6]. Brain tumors categorized into intra-axial and extra-axial in radiologic findings and benign and malignant types in pathologic findings. Based on International Cancer Research Center Report (2012), over 250000 people identified with primary brain tumor in the world which is about two percent of all the number of cancers [7]. Moreover, one quarter of deaths by cancers are caused by metastases to the brain $[8,9]$. The detection of the brain tumors is done by imaging. Different Imaging techniques used to show structural or functional abnormality, like CT scan, MRI, MRS, FMRI, SPECT and PET, and MRI is the most useful and common modality to show the tumors [10]. Manual analysis of brain tumors based on MR images is usually accompanied by numerous problems: different size, shape, texture and consistency of tumors can lead to different results in MR images analysis. Also, the large number of images in each study increases time of data acquisition and analysis [11]. All of the mentioned factors may cause wrong results and delay in decision making [1-4].

One of the most and recent techniques which are used by neurosurgeons or radiologists is extracting tumor's information to show of the tumors [17]. The segmentation of tumors includes extraction, measurement, visualization and presentation of the tumors [5-9]. Correct segmentation of brain tumors can be used for accurate brain modeling and pathologic atlases generation [23,24].

Several techniques have been proposed for the brain tumor segmentation [10,13,16,25-32]. Unfortunately the common practical techniques have some limitations. In recent years, the use of deep learning techniques in image processing application has grown significantly. This study will take a detailed review at the practical aspects of using the deep learning methods in brain tumor segmentation. 


\section{Method}

In this review, we searched IEEE, PubMed, arXiv and Google scholar databases for studies using the combinations of following search keywords: "Brain Tumor Segmentation", "brain tumor detection", "brain MRI segmentation" "deep learning" and "convolutional neural network". Publications in the English language were considered, but we did not impose study design or selection limitations. Review was conducted from relevant articles published between 2010 and 2016.

\section{Brain tumor segmentation}

Image segmentation in the field of computer science, is partitioning of one image into multiple segments to distinguish objects from background [10] This process is represented by sub-images, non-overlapped regions with two properties: Homogeneity within a region, Heterogeneity between the regions [11]. This method is used more meaningful and easier to analyze [12]. Based on various parameters should be considered in any image segmentation task, it has become a complex and multi objective problem [40,42]. Variation in tumor tissue characteristics like shape, size, gray level intensities and localization of tumor may affect on the results of processing [16, 33-36]. In addition, the complicated shape of brain tissues and the structures surrounding the tumor can make it much more sophisticated [37] The other great complexity is when we want to convert the visual information to meaningful regions as a level of semantic understanding of the image contents [43].

With correct brain tumor segmentation, the neurosurgeons are able to understand the location of tumor regions, active tumor tissues, necrotic tissue and edema therefore, it seems that it is a very relevant method for surgical planning. This technique is done by identifying abnormal areas of the brain when compared to normal tissues [13,14,38]. Figure 1 shows some examples of brain tumor MR images with their ground truth segmentation (manually segmented by experts).
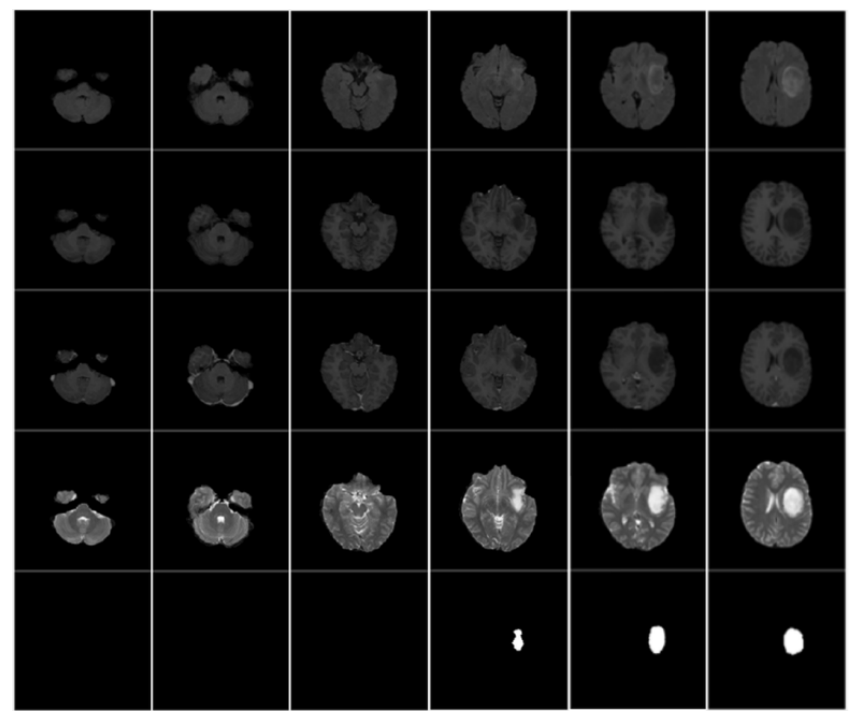

Figure 1. Brain Tumor MRI Scans: top to down: Flair, T1, T1 with contrast, $\mathbf{T} 2$ and ground truth (BRATS)
As we mentioned, the problems in manual analysis of brain tumor segmentation using of image processing techniques are growing [14-16]. Contemplation in the evolution of brain tumor segmentation techniques represent a series of efforts towards achieving the automatic and accurate methods. Meanwhile three generations of image segmentation algorithms have been introduced in the past decades to this goals $[2,15,16$, 26,27,45-54]. The first group of segmentation techniques consist of heuristic methods. Examples of such algorithms are the use of threshold level [17], area growth [18] and separation/merge edge detections $[16,19]$. The general feature of these techniques is the simplicity of their implementation, but the major challenge is the trouble in achieving the desired result when the test conditions have little change compared to their training setting.

The second group of techniques are based on probabilistic models and optimization methods such as: artificial neural networks [20,21], Bayesian models [22], fuzzy clustering [23] and support vector machines [24]. Also, techniques like Gaussian mixture models, linear and non-linear dynamic systems, conditional random fields, maximum entropy (MaxEnt) models, logistic regression, kernel regression and extreme learning machines are in this category [61-64]. These group of techniques are effective in solving simple or wellconstrained problems, but their low modeling capacity cause problems when dealing with complex real-world issues involving natural signals and images processing [65].

The third group of methods seek to achieve the desired result based on the use of higher levels of knowledge. Such knowledge can be inferred from tacit information, rules, and models being created by human or patterns extracted from the bulky data sets. The sample of this generation include Atlasbased segmentation [25] and Deep Learning based methods [26] which is fascinatingly modeled based on the human vision system. Deep learning is a new window to the field of image processing and may revolutionize the medical image processing applications.

\section{Deep Learning}

The human brain's information processing mechanism uses deep architectures to extract complex patterns of information from rich sensor sources. For example, a sound processing and production system in humans is equipped with hierarchical layered structures to convert waveform information to verbal mode and vice versa [68-71]. Also the human vision system is naturally based on a hierarchical structure [72-74]. Modeling of the human brain as the best and most complete samples of natural processors, can guarantee that the most advanced mode of natural signal processing can be achieved by developing by hierarchical learning as in deep learning algorithms [65]. With this idea, deep learning is considered as a hierarchical model which designed to imitate the learning process of the brain's information processing system. 
Deep learning is a hierarchical model, designed with the aim of mimicking the brain's learning process. The deep learning hypothesis is based on the fact that in order to represent a high level of data, a hierarchy of intermediate representations should be generated. Deep learning refers to the learning of multiple levels of representations in order to understand data such as images, audio and text. It includes a kind of machine learning techniques that use a large number of processing layers of linear and nonlinear transforms, with or without supervisor in order to feature extraction, pattern analysis and classification [75-79].

In Deep learning, the first level of representation can be Gabor-like filters, the second level involves identifying lines and angles and the higher levels are gradually moving towards objects and concepts recognition tasks [75-79]. The early idea of deep learning architecture dates back to the 1980s, but due to technical difficulties, their use in practical applications is restricted to the last decade. Some factors make them more popular such as:

- the existence of labeled training collections (some with a total of millions of educational samples);

- more powerful processors along with new generation of GPUs with parallel processing;

- optimized algorithms for training the models [65,76-79].

Deep learning algorithms are capable of learning low-level features and turning them into complex data representation in a hierarchical mode [80]. Unlike other machine learning techniques that feature engineering plays a fundamental role in their success, deep learning methods attempt to learn the hierarchy of features directly from raw data $[81,82]$. Various methods have been developed to implement the deep learning idea include convolution neural networks (CNN), deep belief networks and deep Boltzmann machine. Among these methods, the best technique for exploiting the benefits of multidimensional spatial data such as image, sound and time series is CNN [83].

CNNs are designed to work on multidimensional data, especially images and videos, and has shown its success in many image processing applications $[27,80]$. CNN idea in the field of deep learning, is based on some structural principles derived from a type of the artificial neural networks named multi-layered perceptron (MLP) [28,29]. The CNN functional idea, derived from time delayed neural networks, uses the idea of sharing intra-network weights in order to reduce the network computing time [30]. In this regard, $\mathrm{CNN}$ can be considered as a structure similar to multi-layered neural networks and functionally based on latency networks.

CNNs can be used on raw multidimensional data without preprocessing operations. The Convolutional Neural Network is able to learn the deep general features of multidimensional data such as the image through two basic operations: convolution and sampling.

\section{Convolutional Neural Network (CNN) in brain tumor segmentation}

The CNN structural idea is derived from the multilayered perceptron (MLP) because of the use of layered structure [8486]. Also, the CNN functional idea is based on time delayed neural networks, which uses sharing the intra-network weights in order to reduce the network computations [87]. CNNs can be used on raw multi-dimensional data without preprocessing phase, with a variety of operations, such as convolution, sampling and rectified linear unit, it is able to extract a variety of features from raw data [83].

Like other image processing applications, CNN has been successful in brain tumor segmentation too. Table 1 summarized details of brain tumor segmentation using CNNbased techniques. Also method of deep learning, 2D/3D analysis, implementation tools, datasets, evaluation and important points related to each studies have been covered. In order to investigate the findings, the data in Table 1 represented in distilled form in Figure 1 (a-g).

\section{Discussion}

The details of implementing the CNN algorithms in the brain tumor segmentation presents some tips such as: implementation tools, architectures, input dimensionality, datasets and algorithm evaluation criterions.

The analysis of the tools mentioned in the studies for CNN implementation in brain tumor segmentation shows that the Caffe and the Pylearn's have the major contributions in the implementation. Caffe is implemented in $\mathrm{C}++$ programming language, the Python and MATLAB wrappers are available too [34]. On the other hand, Pylearn is one of the most successful python libraries in the field of deep learning [35]. In addition, there are some platforms like Tensorflow that can be used with the both programming language and are gradually going popular in deep learning applications.

The dominant $\mathrm{CNN}$ architecture in the brain tumor segmentation is Fully Convolutional Network [FCN].

Fully convolutional network $[\mathrm{FCN}]$ is a successful model for image segmentation, which preserves the spatial structure of predictions [36]. In the FCN architecture, a combination of convolution, sampling and deconvolution are used. The point is the distinction between these two models. The first network uses some of the second networks with more limited layers. Indeed, FCN consists of convolution, de-convolution [37], or max-pooling in each layer.

With crossing the input data through the early layers of this architecture, convolution, deconvolution and sampling are used, which compresses information in large number of tinysized feature maps in the middle layers. Subsequently, using deconvolution and sampling, the intermediate feature maps combined together and rebuild the output, which is usually the same size as the input and in the segmented form. 
Table 1. Techniques used in brain tumor MRI images automatic segmentation

\begin{tabular}{|c|c|c|c|c|c|c|c|c|}
\hline & Author & Year & Method & 2D/3D & Implementation & Dataset & Evaluation & Extra notes \\
\hline 1 & Davy et al & 2014 & FCN & & Pylearn2 & BRATS & $\begin{array}{l}\text { Dice } 87 \% \\
\text { PPV } 84 \% \\
\text { Sen } 85 \%\end{array}$ & $\begin{array}{l}\text { N4ITK bias field } \\
\text { correction }\end{array}$ \\
\hline 2 & Zikic et al & 2014 & $\begin{array}{l}\text { CNN with multi-channel } \\
\text { convolutions }\end{array}$ & $2 \mathrm{D}$ & Caffe & BRATS & Accuracy $83 \%$ & $\begin{array}{c}\text { Inhomogeneity correction } \\
\text { in each channel }\end{array}$ \\
\hline 3 & Mikael Agn & 2015 & $\begin{array}{c}\text { Convolutional Restricted } \\
\text { Boltzman Machine + atlas-based } \\
\text { probabilistic model }\end{array}$ & $2 \mathrm{D}$ & MATLAB & BRATS & Dice $83 \%$ & Using atlas \\
\hline 4 & Dvorak \& Menze & 2015 & $\mathrm{CNN}$ & $2 \mathrm{D}$ & Not-detected & BRATS & Dice $83 \%$ & $\begin{array}{l}\text { Local structure prediction } \\
\text { combined with standard } \\
\text { prediction methods }\end{array}$ \\
\hline 5 & $\begin{array}{c}\text { Rao, Shari Sarabi, } \\
\text { \&Jaiswa }\end{array}$ & 2015 & $\mathrm{CNN}$ & $2 \mathrm{D}$ & Caffe & BRATS & Accuracy $67 \%$ & ITK preprocessing \\
\hline 6 & Lyksborg et al & 2015 & $\mathrm{CNN}$ & $2 \mathrm{D}$ & Theano & BRATS & Dice $83 \%$ & $\begin{array}{c}\text { Normalized the intensities } \\
+ \text { patch wised } \\
\text { segmentation }\end{array}$ \\
\hline 7 & Rewari & 2015 & $\mathrm{CNN}$ & & caffe & BRATS & Accuracy $93 \%$ & $\begin{array}{l}\text { N4ITK bias field } \\
\text { correction }\end{array}$ \\
\hline 8 & Y. Pan et al & 2015 & ANN vs CNN & $2 \mathrm{D}$ & & BRATS & Accuracy $66 \%$ & \\
\hline 9 & L. Zhao \& Jia & 2015 & $\begin{array}{l}\text { Triplanar 2D CNN architecture } \\
\text { for 3D voxel classification }\end{array}$ & $3 \mathrm{D}$ & Not-Detected & BRATS & Dice $78 \%$ & \\
\hline 10 & Havaei et al & 2016 & $\mathrm{CNN}$ & $2 \mathrm{D}$ & Pylearn2 & BRATS & Accuracy $81 \%$ & two-pathway architecture \\
\hline 11 & Pereira et al & 2016 & $\mathrm{CNN}$ & $2 \mathrm{D}$ & Theano & BRATS & Dice $78 \%$ & $\begin{array}{c}\text { N4ITK bias field } \\
\text { correction } \\
+ \\
\text { Small } 3 * 3 \text { kernel }\end{array}$ \\
\hline
\end{tabular}

12 Ghafoorian et al 2016

$\mathrm{CNN}$

\section{MRI Scans}

Radboud

University

Nijmegen

Diffusion tensor

and Magnetic

resonance imaging

Cohort

[RUN DMC]

\begin{tabular}{|c|c|c|c|c|c|c|c|c|}
\hline 13 & Chen et al & 2016 & $\mathrm{CNN}$ & $3 \mathrm{D}$ & $\begin{array}{c}\text { Matlab and } \mathrm{C}++ \\
\text { based on Caffe library }\end{array}$ & BRATS & Dice $89 \%$ & Stacked residual modules \\
\hline 14 & Yi et al & 2016 & $\mathrm{CNN}$ & $3 \mathrm{D}$ & Not-Detected & BRATS & Dice $89 \%$ & $\begin{array}{l}\text { a convolutional layer with } \\
\text { pre-defined Difference- } \\
\text { of-Gaussian [DoG] filters }\end{array}$ \\
\hline 15 & Peter D Chang & 2016 & $\mathrm{FCN}$ & $2 \mathrm{D}$ & Matlab & BRaTS & Dice $87 \%$ & $\begin{array}{l}\text { fully convolutional } \\
\text { residual neural network }\end{array}$ \\
\hline
\end{tabular}

15 Peter D Chang 2016

FCN

FCN

3D

Theano

BRATS

Dice $89 \%$

A dual pathway,

11-layers deep, a 3D

16 Kamnitsas 2016

Fully Convolutional

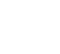

Casamitjana, Puch,

17 Aduriz, Sayrol, \& 2016

Vilaplan

FCN

3D Not-Detected

BRATS

Dice $91.74 \%$

Conditional Random Field

standard normalization $+$

two fully convolutional

3D CNN architectures inspired

N4ITK bias correction $+$

\begin{tabular}{|c|c|c|c|c|c|c|c|c|}
\hline 18 & X. Zhao et al & 2016 & FCN & $2 \mathrm{D}$ & Caffe & BRATS & Dice $80 \%$ & $\begin{array}{c}\text { fully convolutional } \\
\text { network }[\mathrm{FCN}] \text { and } \\
\text { Conditional Random } \\
\text { Fields [CRFs] in a unified } \\
\text { framework }\end{array}$ \\
\hline 19 & Tseng Kuan Lun & 2016 & $\mathrm{FCN}$ & $2 \mathrm{D}$ & Not-Detected & BRATS & Dice $76 \%$ & $\begin{array}{l}\text { Using pre-trained model } \\
\text { as vgg-net }\end{array}$ \\
\hline 20 & Balaji Pandian & 2016 & $\mathrm{CNN}$ & $3 \mathrm{D}$ & Theano & BRATS & Dice $73 \%$ & N4ITK bias correction \\
\hline 21 & $\begin{array}{l}\text { Ramandeep } \\
\text { Randhawa }\end{array}$ & 2016 & $\mathrm{CNN}$ & $2 \mathrm{D}$ & Not-Detected & BRATS & Dice $87 \%$ & $\begin{array}{l}\text { Use two-stage network } \\
\text { training }\end{array}$ \\
\hline 22 & Casamitjana & 2016 & $\mathrm{CNN}$ & $3 \mathrm{D}$ & Not-Detected & BRATS & Dice $89 \%$ & Standard normalization \\
\hline
\end{tabular}




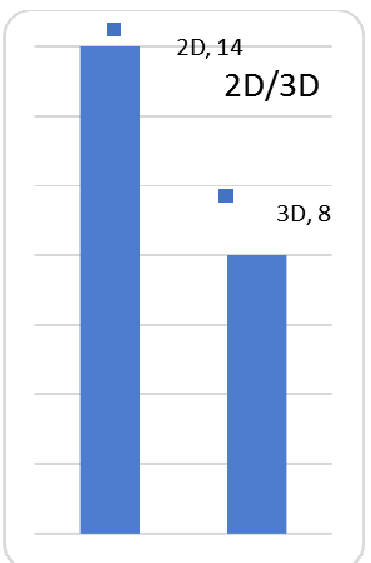

(a) Dimensionality of image processing

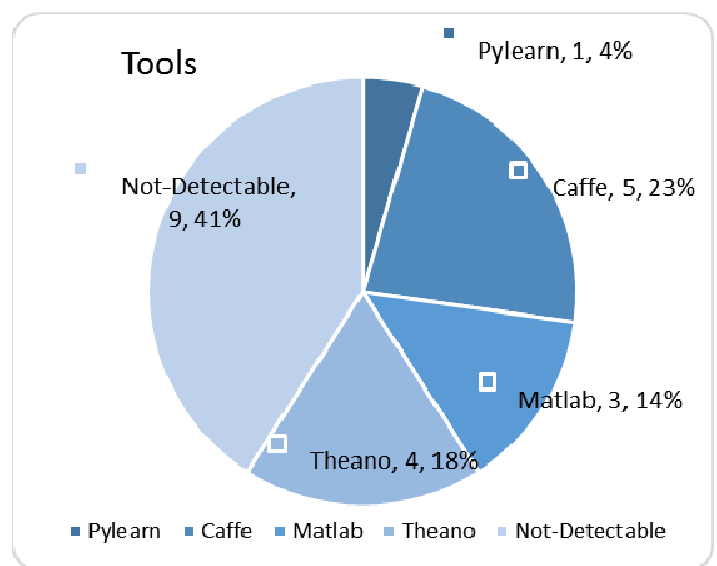

(c) Libraries used for implementation

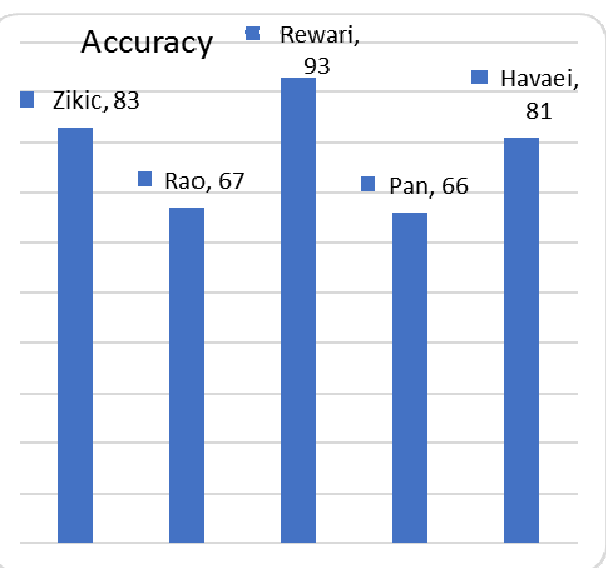

(e) Evaluation based on accuracy

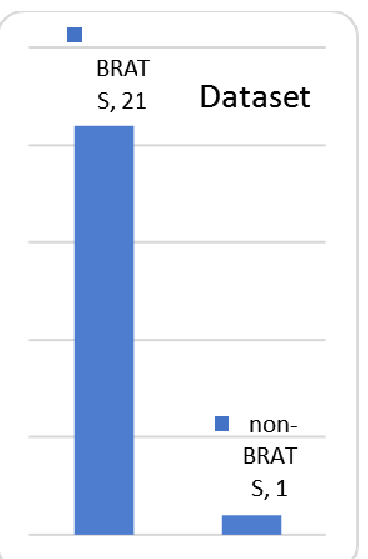

(b) Dataset used in modelling and testing

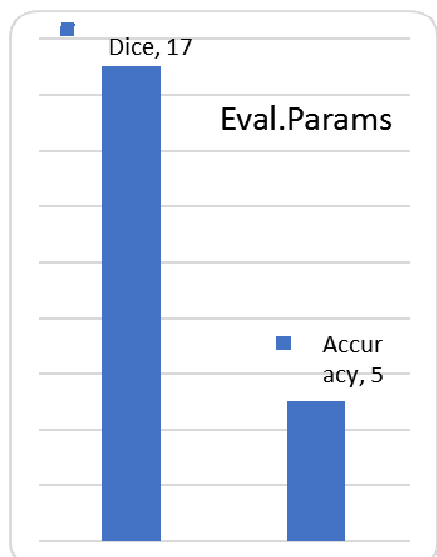

(d) Evaluation parameters

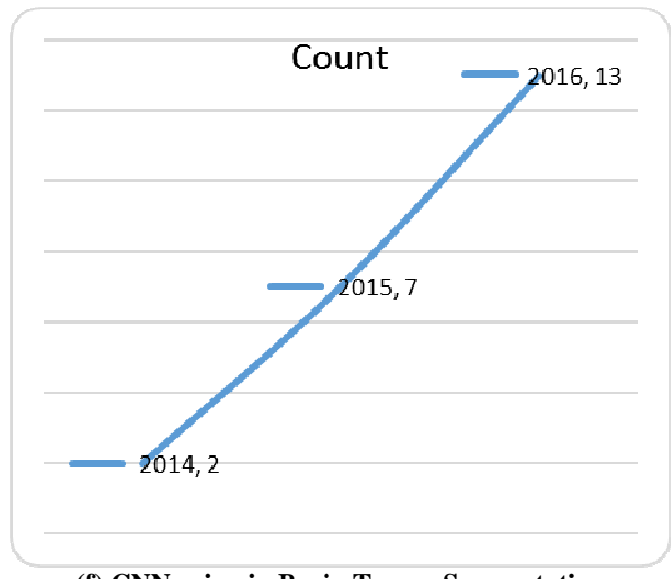

(f) CNN using in Brain Tumor Segmentation

Dice

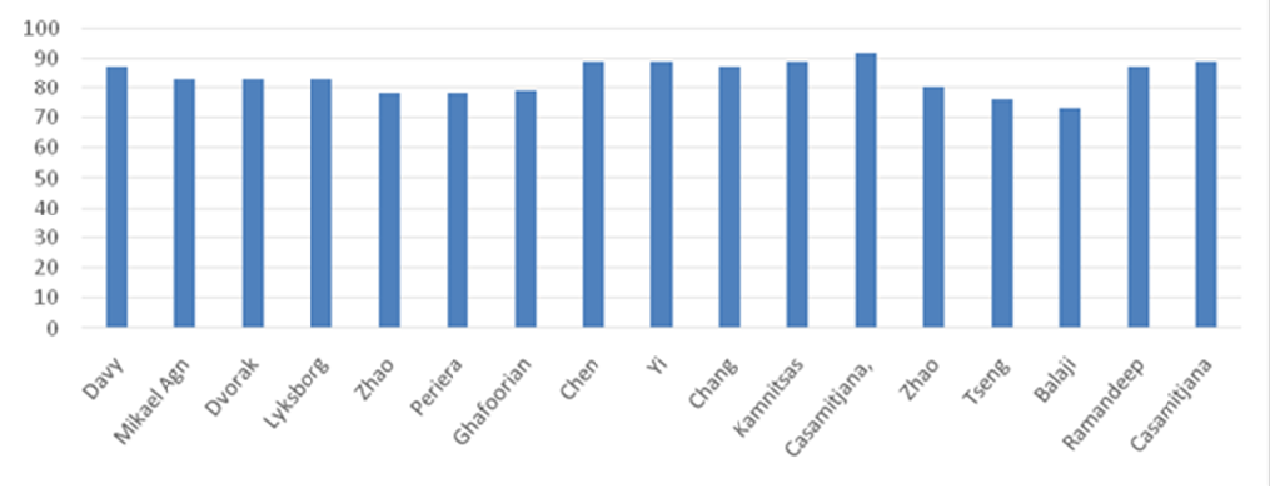

(g) Evaluation based on Dice parameters

Figure 2. Results of review analysis 
This architecture is very suitable for application such as segmentation and therefore has been used frequently in brain tumor segmentation. The studies with two-dimensional images analysis in the literature are more than 3D images ones. Considering that magnetic resonance imaging of the brain tumor is done in 3D form, 3D analysis of image is preferred and emphasized. However, it should be noted that the use of deep learning techniques requires a large number of input samples, in order to fulfill this requirement, each 3D image was converted into dozens of 2D images. Hence, more studies have been done to date on two-dimensional images.

BRATS as the major dataset used in the studies, is a collection of multi-modality brain tumor MR images (T1, T2, T1C and Flair) with their ground truth (manual segmented by experts) [106].

A variety of parameters can be suggested for evaluating segmentation models, and some of them, such as sensitivity, specificity, etc., are mentioned in some studies. But according to the study, the two parameters Dice and Accuracy are the most important parameters for the evaluation of segmentation models and have been used in most of studies in the area of brain tumor segmentation [38-43].

The parameters are calculated as follows. Dice in segmentation evaluation:

Dice $=\frac{2 *|S \cap G|}{|S|+|G|}$

In the above relation, $\mathrm{S}$ is equal to the region segmented by the algorithm and $\mathrm{G}$ is equal to the reference segmentation region.

The Accuracy parameter in the evaluation of segmentation of brain tumor images defined as below:

Accuracy $=\frac{T p+T n}{\text { All pixels }}$

In the above relation, $\mathrm{Tp}$ is the number of tumor pixels identified by the algorithm correctly as a tumorous pixel, and $\mathrm{Tn}$ is the number of non-tumor pixels that are correctly identified by the algorithm as a non-tumorous pixel.

What distinguishes these two parameters, is their power in evaluating the performance of segmentation technique, where Dice focuses specifically on the tumor area, and the Accuracy refers to the overall evaluation of the image. According to the study, some studies have selected the latter for assessing their technique, while the first has been used in most of the studies.

However, using these two parameters (especially Dice) to assess a segmentation technique provides the ability to compare the technique with other studies. As mentioned above, the two parameters are the most commonly used criteria for evaluating fragmentation of brain tumor images.

On the other hand, Dice, can be considered as an appropriate parameter to assess the overlapped areas in the images and can be used as a most useful parameters for evaluation of segmentation [107].
Despite the high interest towards the use of deep learning methods in image processing application, some challenges exist for using them, like:

- Need for bulky datasets:

- This challenge lies in the essence of deep learning, because the philosophy of these techniques depends on the repetition of the feature extraction process. So the analyzes provided by these techniques are generalizable if in the next step, the fine tuning applied on pre-trained networks. In order to solve this challenge, publicly available data sets are being expanded such as BRATS in the case of brain tumor segmentation.

- High computing power required:

- Like the previous challenge, this need is also based on the deep learning paradigm. However, with the advancement of technology in the development of faster hardware and the use of parallel processing architectures, this challenge has been largely overcome.

\section{Conclusion}

Using Dice parameter in future studies can provide a very good measurement for accurate and in detailed comparison of brain tumor segmentation techniques. However, some factors such as geography, race and other demographic parameters can affect practical use of models but BRATS dataset can be used in future studies.. In such situations using BRATS in initial training phase is preferred, then local dataset can be used for fine tuning for better results.

As mentioned earlier, the main feature of deep learning paradigm is independency from engineering processes which leads to extract images from raw data in automatic manner. However, it should be noted that the use of enhancing tools can increase the efficiency of deep learning based methods. As it was shown in the study, the use of preprocessing phase such as N4ITK for bias correction as well as the conditional random field in final processing stage in the $\mathrm{CNN}$ learning can enhance CNN performance in brain tumor segmentation. On the other hand, some strategies in the training of a CNN can increase the performance of the $\mathrm{CNN}$, such as the using of pre-trained models and the use of multi-stage training.

\section{Future perspective}

Deep learning is a revolutionary idea that has created a new paradigm and has gradually shown its success in various image processing applications. The complexity of medical images is high and making decisions about them requires the use of highlevel knowledge and complex feature engineering processes. In such a situation, the use of deep learning techniques will be increased due to their ability to automatic feature extraction. This will create a cycle of usage and success, and we hope in the future to see the application of the deep learning techniques, including the convolutional neural network, in a variety of image processing applications. 


\section{Executive summary}

- Over 250'000 people identified with primary brain tumor in the world which is about two percent of all the cancers, moreover, one quarter of deaths by cancers are caused by metastases to the brain.

- BRATS as the major dataset used in the brain tumor segmentation studies, is a collection of multi-modality brain tumor MR images (T1, T2, T1C and Flair) with their ground truth (manual segmented by experts).

- The detection of brain tumors is done usually by imaging. MRI is the most useful and common modality to show the tumors in the brain MRI scans.

- The most important step of MR image processing is the segmentation of tumors which is used in extraction, measurement, visualization and presentation of the tumors.

- Brain tumor segmentation is a difficult task due to variation in tumor tissue characteristics like shape, size, gray level intensities and location of tumor in the brain.

- Surveying the techniques revealed that three generations of algorithms used in brain tumor segmentation: heuristic methods, probabilistic models and optimization methods and finally methods that use the higher levels of knowledge which can be inferred from tacit information, rules, and models being created by human or patterns extraction.

- Deep learning is a hierarchical model, designed with the aim of mimicking the human brain's learning process and capable of learning low-level features and turning them into complex data representation in a hierarchical mode.

- Convolutional neural network as one of the successful models of deep learning paradigm has been successful in brain tumor segmentation.

- The dominant CNN architecture in the brain tumor segmentation is Fully Convolutional Network [FCN]. In this architecture, a combination of convolution, sampling and deconvolution are used for segmentation.

- The analysis of the dimensions of the input images shows that the number of studies on two-dimensional images of brain scans are larger than 3D images studies.

- Despite the high interest towards the use of deep learning methods in image processing application, some challenges faced for using them like: need for bulky datasets and high computing power.

\section{References}

[1] Priyanka, et al. A review on brain tumor detection using segmentation. International Journal of Computer Science and Mobile Computing. 2013;2(7):48-54.

[2] Maiti I, Chakraborty M. A new method for brain tumor segmentation based on watershed and edge detection algorithms in HSV colour model. in Computing and Communication Systems (NCCCS), 2012 National Conference on. 2012.

[3] Joshi A, et al. An Efficient Tumor Extraction Algorithm using Segmentation of Multimodal Medical Images.

[4] Yu Z, et al. Altered brain anatomical networks and disturbed connection density in brain tumor patients revealed by diffusion tensor tractography. Int J Comput Assist Radiol Surg. 2016;11(11):2007-2019.

[5] Varsha YS, Shyry SP. A Novel Approach for Identifying the Stages of Brain Tumor. International Journal of Computer Trends and Technology (IJCTT) 2014. 10.

[6] Jazayeri SB, et al. Epidemiology of Primary CNS Tumors in Iran: A Systematic. Asian Pacific Journal of Cancer Prevention. 2013;14(6):3979-3985.

[7] Ferlay J, et al. World cancer statistics for the most common cancers 2012. 2012 [cited 2015 16/01/2015.]; Available from: http://www.wcrf.org/int/cancer-facts-figures/worldwide-data.

[8] Gavrilovic IT, Posner JB. Brain metastases: epidemiology and pathophysiology. Journal of neuro-oncology. 2005;75(1):5-14.

[9] Damodharan S, Raghavan D. Combining tissue segmentation and neural network for brain tumor detection. Int. Arab J. Inf. Technol. 2015;12(1): 42-52.

[10] Mehndiratta A, et al. An Introduction to Brain Tumor Imaging, in Tumors of the Central Nervous System, Volume 11. 2014, Springer. p. 3-20.

[11] El-Melegy MT, Mokhtar HM. Tumor segmentation in brain MRI using a fuzzy approach with class center priors. EURASIP Journal on Image and Video Processing, 2014. 2014(1):1-14.

[12] Amsaveni V, Singh NA. Detection of brain tumor using neural network. in Computing, Communications and Networking Technologies (ICCCNT),2013 Fourth International Conference on. 2013.

[13] Schmidt M, et al. Segmenting brain tumors using alignment-based features. in Machine Learning and Applications, 2005. Proceedings. Fourth International Conference on. 2005.

[14] Prajapati SJ, Jadhav KR. Brain tumor detection by various image segmentation techniques with introduction to non negative matrix factorization. Brain. 2015;4(3):600-603. 
[15] Al-Ashwal RH, et al. Digital Processing for Computed Tomography Images: Brain Tumor Extraction and Histogram Analysis. in Math Comput Contemp Sci. 14th International Conference on Mathematical Methods and Computational Techniques in Electrical Engineering (MMACTEE13). 2012.

[16] M Prastawa, et al. A brain tumor segmentation framework based on outlier detection. Med Image Anal. 2004;8(3):275-283.

[17] Ding Z, Wang C, Wang P. Giant Benthic HD Image Feature Extraction and Size Estimation Based on Canny Algorithm. in Proceedings of the 2015 Chinese Intelligent Automation Conference. 2015. Springer.

[18] Wang Y, Ma S. Automatic detection and segmentation of brain tumor using fuzzy classification and deformable models. in Biomedical Engineering and Informatics (BMEI), 2011 4th International Conference on. 2011.

[19] Heiss WD, et al. Multimodality Assessment of Brain Tumors and Tumor Recurrence. J Nucl Med. 2011;52(10):1585-1600.

[20] Moon N, et al. Automatic Brain and Tumor Segmentation. MICCAI proceedings, 2002: p. 372-379.

[21] Wang G, Wang D. Segmentation of Brain MRI Image with GVF Snake Model. in Pervasive Computing Signal Processing and Applications (PCSPA), 2010 First International Conference on. 2010.

[22] Bara S, et al. A robust approach for the detection of brain tumors by variational b-spline level-set method and brain extraction. in Multimedia Computing and Systems (ICMCS), 2014 International Conference on. 2014.

[23] Logeswari T, Karnan M. An improved implementation of brain tumor detection using segmentation based on soft computing. Journal of Cancer Research and Experimental Oncology. 2009;2(1)::006-014.

[24] Toga AW, et al. Probabilistic approaches for atlasing normal and disease-specific brain variability. Anatomy and embryology. 2001; 204(4):267-282.

[25] Shanthi U, et al. An Automated Computer Aided System for Tumor Detection in Brain. International Journal of Innovative Research in Computer and Communication Engineering. 2015;3(2):84-88.

[26] Kumar M, Mehta KK. A Texture based Tumor detection and automatic Segmentation using Seeded Region Growing Method. ijta, 2015;2(4):855-859.

[27] Fazli S, Nadirkhanlou P. A Novel Method for Automatic Segmentation of Brain Tumors in MRI Images. arxiv, 2013.

[28] Al-Tamimi MSH, Sulong G. Tumor brain detection through MR images: a review of literature. Journal of Theoretical and Applied Information Technology. 2014;6(2):387-403.

[29] Malviya AM, Joshi AS. Gabor Wavelet Approach for Automatic Brain Tumor Detection. International Journal of Emerging Technology and Advanced Engineering. 2014;4(4): 826-831.

[30] Goswami S, Bhaiya LKP. A hybrid neuro-fuzzy approach for brain abnormality detection using GLCM based feature extraction. in Emerging Trends in Communication, Control, Signal Processing \& Computing Applications (C2SPCA), 2013 International Conference on. 2013.

[31] Ibrahim WH, Osman AAA, Mohamed YI. MRI brain image classification using neural networks. in Computing, Electrical and Electronics Engineering (ICCEEE), 2013 International Conference on. 2013.

[32] Phooi Yee L, Voon FCT, Ozawa S. The detection and visualization of brain tumors on T2-weighted MRI images using multiparameter feature blocks. in Engineering in Medicine and Biology Society, 2005. IEEE-EMBS 2005. 27th Annual International Conference of the. 2005.

[33] Havaei M, et al. Brain tumor segmentation with deep neural networks. Medical image analysis. 2017;35:18-31.

[34] Sachdeva J, et al. A novel content-based active contour model for brain tumor segmentation. Magnetic resonance imaging. 2012;30(5):694-715.

[35] Aswathy SU, Dhas GGD, Kumar SS. A survey on detection of brain tumor from MRI brain images. in 2014 International Conference on Control, Instrumentation, Communication and Computational Technologies (ICCICCT). 2014.

[36] Xuan X, Liao Q. Statistical structure analysis in MRI brain tumor segmentation. in Image and Graphics, 2007. ICIG 2007. Fourth International Conference on. 2007. IEEE.

[37] Chen D. Interactive Brain Tumor Segmentation with Inclusion Constraints. 2016, The University of Western Ontario.

[38] Upadhyay N, Waldman A. Conventional MRI evaluation of gliomas. Br J Radiol. 2011;84(Spec No 2):S107-11.

[39] Sheikh A, Krishna R, Dutt S. Energy Efficient Approach for Segmentation of Brain tumor Using Ant Colony Optimizationll. International Journal of Computer Technology and Electronics Engineering (IJCTEE) Volume. 1.

[40] Dass R, Devi S. Image segmentation techniques 1. 2012.

[41] Kumar D. Review on different Techniques of Image Segmentation using MATLAB. 2017.

[42] Chin-Wei B, Rajeswari M. Multiobjective Optimization Approaches in Image Segmentation-The Directions and Challenges. Int. J. Advance. Soft Comput. Appl, 2010. 2(1).

[43] Arbeláez PA, Cohen LD. Energy partitions and image segmentation. Journal of Mathematical Imaging and Vision. 2004;20(1):43-57.

[44] Costin H. Recent trends in medical image processing. Computer Science. 2014;22(2):65. 
[45] Withey D, Koles Z. A review of medical image segmentation: methods and available software. International Journal of Bioelectromagnetism. 2008;10(3):125-148.

[46] Sachin N, et al. Brain Tumor Detection Based On Bilateral Symmetry Information. Int. Journal of Engineering Research and Application. 2013;3(6):430-432.

[47] Constantin AA, Bajcsy BR, Nelson CS. Unsupervised segmentation of brain tissue in multivariate MRI. in Biomedical Imaging: From Nano to Macro, 2010 IEEE International Symposium on. 2010.

[48] Kobashi S, et al. Adaptive Brain Tissue Classification with Fuzzy Spatial Modeling in 3T IR-FSPGR MR Images. in Automation Congress, 2006. WAC '06. World. 2006.

[49] Yu CP, et al. 3D blob based brain tumor detection and segmentation in MR images. in Biomedical Imaging (ISBI), 2014 IEEE 11th International Symposium on. 2014.

[50] D Jude hemanth, Selvathi D, Anitha J. Effective Fuzzy Clustering Algorithm for Abnormal MR Brain Image Segmentation. IEEE International Advance Computing Conference. 2009;6(7):609-614.

[51] Karnan M, Selvanayaki K. Improved implementation of brain MR image segmentation using Meta heuristic algorithms. in Computational Intelligence and Computing Research (ICCIC), 2010 IEEE International Conference on. 2010.

[52] Idrissi N, Ajmi FE. A hybrid segmentation approach for brain tumor extraction and detection. in Multimedia Computing and Systems (ICMCS), 2014 International Conference on. 2014.

[53] Charutha S, Jayashree MJ. An efficient brain tumor detection by integrating modified texture based region growing and cellular automata edge detection. in Control, Instrumentation, Communication and Computational Technologies (ICCICCT), 2014 International Conference on. 2014.

[54] Vijay J, Subhashini J. An efficient brain tumor detection methodology using K-means clustering algoriftnn. in Communications and Signal Processing (ICCSP), 2013 International Conference on. 2013.

[55] Mustaqeem A, Javed A, Fatima T. An efficient brain tumor detection algorithm using watershed \& thresholding based segmentation. International Journal of Image, Graphics and Signal Processing. 2012;4(10):34.

[56] Cuadra MB, et al. Atlas-based segmentation of pathological MR brain images using a model of lesion growth. IEEE transactions on medical imaging. 2004;23(10):1301-1314.

[57] Shen S, et al. MRI fuzzy segmentation of brain tissue using neighborhood attraction with neural-network optimization. IEEE transactions on information technology in biomedicine. 2005;9(3):459-467.

[58] Corso JJ, et al. Efficient multilevel brain tumor segmentation with integrated bayesian model classification. IEEE transactions on medical imaging. 2008;27(5):629-640.

[59] Hall LO, et al. A comparison of neural network and fuzzy clustering techniques in segmenting magnetic resonance images of the brain. IEEE transactions on neural networks. 1992;3(5):672-682.

[60] Bauer S, Nolte L-P, Reyes M. Fully automatic segmentation of brain tumor images using support vector machine classification in combination with hierarchical conditional random field regularization. Medical image computing and computer-assisted intervention-MICCAI 2011, 2011: p. 354-361.

[61] Aslan Ö, et al. Convex two-layer modeling. in Advances in Neural Information Processing Systems. 2013.

[62] Cho Y, Saul LK. Kernel methods for deep learning. in Advances in neural information processing systems. 2009.

[63] Deng L, et al. Use of kernel deep convex networks and end-to-end learning for spoken language understanding. in Spoken Language Technology Workshop (SLT), 2012 IEEE. 2012. IEEE.

[64] Vinyals O, et al. Learning with recursive perceptual representations. in Advances in Neural Information Processing Systems. 2012.

[65] Huertas-Company $\mathrm{M}$, et al. A catalog of visual-like morphologies in the 5 CANDELS fields using deep-learning. arXiv preprint arXiv:1509.05429, 2015.

[66] Bauer S, et al. Atlas-based segmentation of brain tumor images using a Markov random field-based tumor growth model and nonrigid registration. in Engineering in Medicine and Biology Society (EMBC), 2010 Annual International Conference of the IEEE. 2010. IEEE.

[67] Pereira S, et al. Brain tumor segmentation using convolutional neural networks in MRI images. IEEE transactions on medical imaging. 2016;35(5):1240-1251.

[68] Baker JM, et al. Developments and directions in speech recognition and understanding, Part 1 [DSP Education]. IEEE Signal Processing Magazine. 2009;26(3):75-80.

[69] Baker J. et al. Updated MINDS report on speech recognition and understanding, Part 2. 2009. Institute of Electrical and Electronics Engineers.

[70] Deng L. Computational models for speech production, in Computational models of speech pattern processing. 1999, Springer. p. 199-213.

[71] Deng L. Switching dynamic system models for speech articulation and acoustics, in Mathematical Foundations of Speech and Language Processing. 2004, Springer. p. 115-133. 
[72] George, D., How the brain might work: A hierarchical and temporal model for learning and recognition. 2008, Citeseer.

[73] Bouvrie JV. Hierarchical learning: Theory with applications in speech and vision. 2009, Citeseer.

[74] Poggio T. How the brain might work: The role of information and learning in understanding and replicating intelligence. Information: Science and Technology for the New Century, 2007: 45-61.

[75] Deng L, Yu D. Deep Learning. Signal Processing. 2014;7:3-4.

[76] Hinton GE, Osindero S, The Y-W. A fast learning algorithm for deep belief nets. Neural computation. 2006;18(7):1527-1554.

[77] Mohamed A-r, Hinton GE. Phone recognition using Restricted Boltzmann Machines. in ICASSP. 2010.

[78] Le QV, et al. Learning hierarchical invariant spatio-temporal features for action recognition with independent subspace analysis. in Computer Vision and Pattern Recognition (CVPR), 2011 IEEE Conference on. 2011. IEEE.

[79] Vincent $P$, et al. Stacked denoising autoencoders: Learning useful representations in a deep network with a local denoising criterion. Journal of Machine Learning Research. 2010;11: 3371-3408.

[80] Zhou X, et al. Product Image Search with Deep Attribute Mining and Re-ranking. in Pacific Rim Conference on Multimedia. 2016. Springer.

[81] Bengio Y. Learning deep architectures for AI. Foundations and trends® in Machine Learning. 2009;2(1):1-127.

[82] Ciresan D, et al. Deep neural networks segment neuronal membranes in electron microscopy images. in Advances in neural information processing systems. 2012.

[83] Vo DM, Le TH. Deep generic features and SVM for facial expression recognition. in Information and Computer Science (NICS), 2016 3rd National Foundation for Science and Technology Development Conference on. 2016. IEEE.

[84] LeCun Y, et al. Gradient-based learning applied to document recognition. Proceedings of the IEEE, 1998. 86(11):2278-2324.

[85] Huang FJ, LeCun Y. Large-scale learning with svm and convolutional for generic object categorization. in Computer Vision and Pattern Recognition, 2006 IEEE Computer Society Conference on. 2006. IEEE.

[86] Goodfellow I, Bengio Y, Courville A. Deep Learning. MIT Press; 2016.

[87] Waibel A, et al. Phoneme recognition using time-delay neural networks. Acoustics, Speech and Signal Processing, IEEE Transactions on, 1989:37(3): 328-339.

[88] Davy A, et al. Brain tumor segmentation with deep neural networks. 2014.

[89] Zikic D, et al. Segmentation of brain tumor tissues with convolutional neural networks. Proceedings MICCAI-BRATS, 2014:36-39.

[90] Agn M, et al. Brain tumor segmentation by a generative model with a prior on tumor shape. Proceeding of the Multimodal Brain Tumor Image Segmentation Challenge, 2015: 1-4.

[91] Dvorak P. Menze B. Structured prediction with convolutional neural networks for multimodal brain tumor segmentation. Proceeding of the Multimodal Brain Tumor Image Segmentation Challenge, 2015:13-24.

[92] Rao V. Shari Sarabi M, Jaiswal. Brain tumor segmentation with deep learning. MICCAI Multimodal Brain Tumor Segmentation Challenge (BraTS), 2015: 56-59.

[93] Lyksborg M, et al. An ensemble of 2D convolutional neural networks for tumor segmentation. in Scandinavian Conference on Image Analysis. 2015. Springer.

[94] Rewari R. Automatic Tumor Segmentation from MRI scans. Stanford University.

[95] Pan Y, et al. Brain tumor grading based on neural networks and convolutional neural networks. in 2015 37th Annual International Conference of the IEEE Engineering in Medicine and Biology Society (EMBC). 2015. IEEE.

[96] Zhao L, Jia K. Deep feature learning with discrimination mechanism for brain tumor segmentation and diagnosis. in 2015 International Conference on Intelligent Information Hiding and Multimedia Signal Processing (IIH-MSP). 2015. IEEE.

[97] Havaei M, et al. Brain tumor segmentation with deep neural networks. Medical Image Analysis, 2016.

[98] Ghafoorian M., et al. Location Sensitive Deep Convolutional Neural Networks for Segmentation of White Matter Hyperintensities. arXiv preprint arXiv:1610.04834, 2016.

[99] Chen H, et al. VoxResNet: Deep Voxelwise Residual Networks for Volumetric Brain Segmentation. arXiv preprint arXiv:1608.05895, 2016.

[100] Yi D. et al. 3-D Convolutional Neural Networks for Glioblastoma Segmentation. arXiv preprint arXiv:1611.04534, 2016.

[101] Chang PD. Fully Convolutional Deep Residual Neural Networks for Brain Tumor Segmentation, in Brainlesion: Glioma, Multiple Sclerosis, Stroke and Traumatic Brain Injuries: Second International Workshop, BrainLes 2016, with the Challenges on BRATS, ISLES and mTOP 2016, Held in Conjunction with MICCAI 2016, Athens, Greece, October 17, 2016, Revised Selected Papers, A. Crimi, et al., Editors. 2016, Springer International Publishing: Cham. p. 108-118.

[102] Kamnitsas K, et al. DeepMedic on Brain Tumor Segmentation. Athens, Greece Proc. BRATS-MICCAI, 2016.

[103] Casamitjana A, et al. 3D convolutional networks for brain tumor segmentation. Proceedings of the MICCAI Challenge on Multimodal Brain Tumor Image Segmentation (BRATS), 2016:65-68. 
[104] Zhao X, et al. A deep learning model integrating FCNNs and CRFs for brain tumor segmentation. arXiv preprint arXiv:1702.04528, 2017.

[105] Lun T, Hsu W. Brain tumor segmentation using deep convolutional neural network. Proceedings of BRATS-MICCAI, 2016.

[106] MICCAI. about MICCAI. 2016 [cited 2016; Available from: http://www.miccai.org/organization].

[107] Veta M, et al. Automatic nuclei segmentation in H\&E stained breast cancer histopathology images. PloS one. 2013;8(7): e70221.

[108] Kang J. et al. Neuron sparseness versus connection sparseness in deep neural network for large vocabulary speech recognition. in 2015 IEEE International Conference on Acoustics, Speech and Signal Processing (ICASSP). 2015. IEEE.

[109] Kleć M, Koržinek D. Pre-trained deep neural network using sparse autoencoders and scattering wavelet transform for musical genre recognition. Computer Science. 2015;16(2): 133-144.

[110] Oyallon E, Mallat S, Sifre L. Generic deep networks with wavelet scattering. arXiv preprint arXiv:1312.5940, 2013.

[111] Hassairi S, Ejbali R, Zaied M. Supervised image classification using Deep Convolutional Wavelets Network. in Tools with Artificial Intelligence (ICTAI), 2015 IEEE 27th International Conference on. 2015. IEEE. 